\title{
Nanotechnology and its partnership with synbiotics
}

\begin{abstract}
In the realm of nanotechnology, there are no barriers aside from a researcher's imagination that can prevent the field from being a reputable force. The emergence of nanomedicine is an actual benefit in the increased interest placed within the food sector. As the field evolves, it is in a constant state of improvement. Researchers seek for innovative ways to combine the fundamentals of Nano-measurement with the emerging importance of colon health. Nanotechnology is becoming a valuable source of supportive help to the once known golden standards of gastroenterology. The once preventative measures used for colon carcinomas, ulcerative colitis, and Celiac Disease and an option for post treatment bacterial and viral enteritis, has gotten the sense of melioration in the technology. There is an importance in understanding the microbial flora of the gut, and the efforts to maintain it and with promising results; nanotechnology is becoming increasingly important for the food sector.
\end{abstract}

Keywords: Colon health, Prebiotics, Nanotechnology, Probiotics, Human milk Oligosaccharides, Lactobacillus, Microbiota, Synbiotics, Colorectal carcinoma
Volume 6 Issue I - 2017

\author{
Dercas Caneus \\ University of Science Arts and Technology, USA
}

Correspondence: Dercas Caneus, University of Science Arts, and Technology, USA, Tel 9549931982,

Email dercas.caneus@usat.edu

Received: July 27, 2017| Published: August 08, 2017
Abbreviations: TNF- $\alpha$, Tumor necrotizing Factor-Alpha; LPS, Liposaccharide; GalN, Galactosamine, IL-1 $\beta$, Interleukin-1 Beta; HMO, Human Milk Oligosaccharide; GOS, Galactooligo Saccharides; ASD, Autism Spectrum Disorder; NO, Nitric Oxide; MIA, Maternal Immune Activation; DBM, Dibenzoyl-Methane; PET, Probiotic Encapsulation Technology; CRC, Colorectal Cancer; NP, Nanoparticles; FGNP, Food Grade Nanoparticles; AOM, Azoxymethane; ACF, Aberrant Crypt Foci

\section{Introduction}

A field that has become increasingly of interest to medical researchers and based on particle size is nanotechnology, which has evolved and involved every facet of science, engineering, and technology. For this reason, nanotechnology has become a central focus in providing medical treatment advancement, through creating medicine with a unique perspective conducted at scales less than 100 nanometers. To understand the relevance of a nanometer, one must appreciate its size. There are approximately 2.54 million nanometers in an inch; a billionth of a meter and a single sheet of a newspaper are each 100,000 nanometers in thickness. ${ }^{1}$ Nanotechnology significantly relies on this scale to perform manipulation of molecules to influence the desired effect of both a compound and its cellular target. It is this rationale that has had the intrinsic value in the attempts to develop nano-treatments in gastroenterology. When diet inadvertently influences pre-carcinogen and mutagenic metabolites, it's the gastrointestinal mucosal cells that cause their activation. ${ }^{2}$ It is the advent of nanomedicine that confers it be appropriate to forge a union with the known practices of Prebiotics, Probiotics, and Synbiotics in creating an optimal environment within the gastrointestinal tract; thus become the most valuable approach.

\section{Nanotechnology}

Potential nano-carriers provide new opportunities for the food industry, based on delineation of their physical properties such as size, composition, morphology, surface area and its charge, to create nanoliposomes, nanoemulsions, nanoparticles, and nanofibers. ${ }^{3}$ It is the vast array of structural arrangements that make the nanosystems versatile. The FGNP fall into three capital categories: inorganic, surface functionalized materials and organic engineered nanomaterials. Of note "top-down," "bottom-up," biomimetic, and functional assemblies have been used in the administration of the nanomedicine. During the formation of the viral associative nanomaterials, reversible disassembly and reassembly in response to ionic strength, temperature, and $\mathrm{pH}$ may occur. Upon removal, the nanomaterial formed from viruses undergoes genomic RNA deletion, and the viral capsid, as the center of the vaccine or therapy, may then be utilized to encapsulate potential functional material. In the development of nanomedicine, these physical properties are exploited to play a fundamental role in cellular uptake, intracellular distribution, and possible accumulation within an organism's system. Surprisingly, the world consists of nano-materials that already occur in nature. Nature has formed nanotubes and nanoparticles, which present them in a variety of conformations, such as sea spray, smoke, or volcanic ash. Nanotechnology has been beneficial in the overall progression of medical science and has the potential to provide future treatments, and furthering currently available therapies. Encapsulated drugs do not exhibit the traditional pharmacokinetic profiles found in free drugs. In the formulation of FGNP requires a strategy, because the nanocarriers dictate the drugs overall distribution and efficacy, it has the luxury of directly interacting with the cell membrane and intracellular structures at ease.

\section{Colon health}

\section{Probiotics}

Probiotics are the more widely known culprit of a healthy digestive tract, known to be the "good" bacteria; it consists of "live microorganisms" found in various fermented dairy and non-dairy products, whose job is to prevent the overgrowth of harmful bacteria hence promoting healthy gut microflora. ${ }^{4}$ Introducing probiotics into a person's diet has other benefits outside the host's gut, as an introduction of empirical therapy or prophylaxis for common urogenital infections. ${ }^{5}$ It is one of the gold standards, along with medication and supplements for replenishing the gut from depletion of its microflora. Dysbiosis, resulting from an assault received from the long-term use of antibiotics, disease processes of the gastrointestinal tract such as Colon Carcinoma, Ulcerative colitis, IBS, Crohns Disease, Inflammatory Bowel Disease, Celiac Disease, and Traveler's Diarrhea, can leave the gut susceptible, and can benefit from its implementation. Research has supported probiotics to have anti-inflammatory properties beneficial for the entire body, improve 
post menopausal symptoms, to address lactose intolerance, and to lower cholesterol levels, ${ }^{6}$ as one study stated by treating rats with $L$. plantarum with nanoparticle Tellurium, observed a reduction in the levels of the triglycerides. ${ }^{7}$

There are different strains and species of probiotics that companies manufacture. The most common species available on the market are Lacto bacillus, Bifido bacterium, Bacteroides, Saccharomyces, and Streptococcus ${ }^{8}$ typically marked on dietary products containing "live or active cultures." Another great source to find probiotics is sourdough bread, kefir, sauerkraut, miso soups; it is the fermentation process that produces the valuable bacterium. Assuring the quality of the probiotic is crucial to its efficacy. ${ }^{9}$ There is a criterion that has been developed to constitute a food product as a probiotic; it must contain live microorganisms and be in adequate amounts to be considered a benefit to the host when administered. ${ }^{10}$ Probiotics, a known formidable opponent in the health of gastrointestinal tract, has its limitations: they are delicate to heat, stomach acid, and time sensitive. This impediment causes the elimination of a significant number before the probiotics have the opportunity to fulfill its purpose, which is a disadvantage for the microorganisms. Even looking for unpasteurized dietary products would help combat this problem but isn't the only solution. Thus developing a system of delivery is essential for the stronger, healthier gut.

\section{Prebiotic sugars}

The less unknown, yet just as important collaborator in the growth of the microflora within the gut is prebiotics. Prebiotics are non-living sugars; carbohydrates typically a specialized plant fiber that cannot be digested by the human body ${ }^{11}$ that the natural microflora within the colon and probiotics feed on; this promotes the growth of the microbiota because it is its "food" source. This synergistic effect is known as synbiotics, where the prebiotics is the food source for the probiotics. The characteristics of prebiotics are heat labile, cold, acid and time resistant, which enables the stimulation of proliferation of commensal microbial genus within the gut for health benefits to the host. Some prebiotics is artichokes, kefir, oatmeal, red wine, honey, $\mathrm{HMO}^{12}$ and legumes. A study conducted showed GOS, which is similar to HMO when added to an infant's diets revealed its gut health was analogous to that of breast feed infants. ${ }^{13}$ The study took 371 full term infants, who'd been breastfed initially for two weeks. Then randomly selected within the sample group to be supplemented with GOS, then followed-up in 3 months. The study revealed the $\mathrm{pH}$ had decreased, and the stool frequency had significantly increased; GI conditions that are known to promote "good" colon health.

\section{Application to nanotechnology}

There is research that has shown the benefits of combining the technology and current practices to promote a healthier colon. With having regular bowel movements, it reduces the effects of exotoxins released in the gut, which in turn will reduce the incidences of precancerous polyps and colon carcinoma with idiopathic etiology. Nanotechnology has the capacity for delivering the catalyst to it intended environment, extending it time, and allowing the prebiotic to help feed and aide in a flourishing gut flora. This advent technology becomes a great benefit to chronic digestive disorders, and inflammatory bowel disease. Lest not forget the recent exploration of gut and microbiome health and its relation to neuroscience and physical health. Evidence has shown increased level of stress hormones from the response of anxiety, depression, and gastrointestinal symptoms were associated with autism and had correlations between changes observed in the colon's micro flora, of those who did not suffer from neurological ailments. ${ }^{14}$ A possible linkage between ASD and gastroenterology, those with autism have shown dysbiosis; a disruption in the balance of the microbiota community composition and abnormalities in the gut, with increased gastrointestinal permeability and dysmotility. A modeling MIA offspring showed the linkage of increased risk of ASD and increased inflammatory factors found in the maternal blood, amniotic fluid, and placenta. When given the probiotic B. fragilis, it was found to correct the intestinal permeability in the MIA offspring. ${ }^{15}$ The novel trend is to design healthy food with the use of nanoscience and its desired characteristics and properties to create a food grade particle unique to the nanoscale that becomes a benefit for the overall well being of its host.

\section{Anti-inflammatory effects found in Probiotics}

A study conducted with the L.casei showed to reduce cytokine effects seen in rats with acute liver failure. For thirty days researchers orally administered L.casei, serum, liver, and intestinal samples were taken 8 hours after the administration of LPS and GalN. Results of the challenge showed an increased production of stress hormones, and endotoxins, like TNF- $\alpha$, IL- $1 \beta$, and NO in rats who were only treated with dexamethasone, while those treated with L.casei displayed a reduction in myeloperoxidase activity, prevention of intestinal injury and reduced the expression of IL-1 $\beta .{ }^{16}$ In this world, we have further paced ourselves away from concerns such as infectious diseases, but with a discovery of antibiotic-resistance pathogen conventional therapies are no longer sufficing; an exacerbation of this matter was brought about by the notion of established pathogen resurfacing with variations of its former shell. ${ }^{17}$ Medical research seeks a more improve therapy for infections on the rise that affects the intestinal tract.

\section{Encapsulation of Probiotic bacteria}

One of the current methodologies for FGNP that has been useful in the entrapment and delivery of the heat-sensitive probiotics is PET. PET, a colloidal delivery system whose concept is encapsulating a bacterium in a gel-like suspension, then packing the substance into a miniature-sized final product that has the enhanced power of functionality and the ability to control the release of the core, increasing the stability of the pharmaceutical-grade nutrient. ${ }^{18,19}$ It provided access to the probiotic to bypass the unsuitable environment such as the acid and bile-rich stomach. A study formed a goal to improve oral bioavailability and improve the efficacy of chemo-preventive compound DBM, through nanoemulsion. The nano-formation was from water, oil, and emulsifier under high speed and high-pressure homogenization, ${ }^{20,21}$ this compound is known to have anticancer effects, and getting through the harsh environment of the gastrointestinal tract was of extreme importance. Further studies reported the utilization of microencapsulation of calcium alginate-starch and the incorporation of Hi-Maize increased its survival of the probiotic and were further enhanced the introduction of the cryoprotectant glycerol; protecting it in temperatures of 20 degrees Celsius and slowing the effects of acid production and traveling through the stomach without any degradation. ${ }^{22}$ With the market demand increasing for better-stabilized methods of deliveries and specific request towards lactose intolerant and vegetarian-friendly products, it called for an increase to cerealbased probiotics. Bioavailability of a probiotic has benefited from nanoscience, being possible to design fermented beverages with synbiotic properties. ${ }^{23,24}$ Studies have suggested the environment of the gastrointestinal tract, has an increased association to the incidences of polyps and colon cancer. Requiring for a plan to devise tools and methods to circumvent the dilemma is necessary. One study reported in rats that the stimulation growth of bifidobacteria leads to the inhibition colon cancer formation, attributed to lowering $\mathrm{pH}$, and antitumor properties from the bacterium. ${ }^{25}$ Such properties inhibited 
the growth of E.coli, and Clostridia in turn producing modulations to pre-carcinogens of the bacterium. Coupling nanotech with an effective therapy may help overcome the challenges encountered in colon related antineoplastic drug development and the prevention of tumor resistance from chemotherapy. ${ }^{26}$

\section{Nanotechnology and Synbiotic A Protection against Colorectal Cancer}

Nanotechnology has been used steadfastly in imaging involving the diagnosis of cancer. However, with the trend looking towards synbiotics; Prebiotics and Probiotics can exert health benefits when consumed, acting as gut modulators, and with nanotechnology in tow, it is progressively taking hold the field. As seen in evidence-based practices, studies have shown reducing the risk of colon carcinogenesis; impart to the fatty acid chains created in the fermentation process to form probiotics. ${ }^{27}$ The synergistic effect exerts alternations to the tumor expressing genes. ${ }^{28}$ A randomized, controlled, comparative study utilize heat -killed L.casei in 228 patients with stage III cervical cancer, it reports antitumor effects caused by the macrophage activation by the L.casei. The study combined radiation therapy and the introduction of LC9018, and its effectiveness as an adjuvant immunotherapy for cervical cancer. ${ }^{29}$ When rats treated with AOM induced colonic, a known carcinogenic it reports that the skeletal cell wall of mutagens found adherence factors of bifidobacteria, thus minimizing the carcinogenic absorption effects into the intestinal lumen. ${ }^{25,30}$ Nanotechnology can enhance the reach a drug has, solely based on it is size. The slight ease found in interaction, and ability to evade clearance can improve efficaciousness of a drug. A group aimed to encapsulate the known cancer drug Oxaliplatin, to help with efficacy and cytotoxicity by using NP. The study reported positive result from the nanosystem in a xenograft model to efficiently target tumor cells. The NP particles where $\mathrm{pH}$ sensitive and were design to release it chemotherapies, once it passes through the acidic environment of the stomach to solely target the intestinal tract. In vivo, the nanoparticles prove to reduce the burden from the tumor cells and mortality of an orthotropic mouse with CRC. ${ }^{31}$

\section{Conclusion}

It is still an emerging field, with obstacles seen in the current market due to current mindset of consumers because of a market innodated with misleading labels of used and unused species. The commercialization of Prebiotics and Probiotics has seen minuscule movement in the field of food-nanotechnology regardless of research supporting the health claims has been a long and tedious process from lab to consumer. Which is important to institute regulations and standards uniformly across the countries that will allow, evidence based claims to move forward opposed those who are presenting factless claims. ${ }^{32}$ Thus continuing the research must go on. The future of FGNP depends on the standards set.

The advancements seen from nanotechnology within the last 20 years, has leaped the human body and the world forward by creating a limitless environment of improvement and innovation. An area that has seen such a benefit is the gastrointestinal tract's health; from an infant in helping with colic to adults with the various digestive maladies. With drug delivery having the ability to be combined with nanotechnology, synbiotics as a concomitant with cytotoxic and anti-vascular chemotherapies makes this assembly an attractive approach for the treatment of gastrointestinal ailments. With statistics and research supporting the benefit of a happy gut in the prevention of carcinomas, the possible balance between the gut bacteria-brain links; such as depression, autism, and anxiety, nanotechnology can dramatically change the course of food technology, propel forward. The application of nanotechnology has, therefore, an immense potential in overcoming or circumventing the extra- and intracellular mechanisms associated with drug resistance. With nanotechnology partaking in the advancement of the field of gastroenterology, the "Golden Standard" has evolved from solely the use of imaging and colonoscopy, to find a formidable partnership in nanotechnology.

\section{Acknowledgements}

No financial assistance for the research work.

\section{Conflicts of interest}

None.

\section{References}

1. What Is Nanotechnology?

2. Wan Fen F, Strobel HW. Activation of Carcinogens and Mutagens by Rat Colon Mucosa. Cancer Res. 1978;38:2939-2944.

3. Weiss J, Takhistov P, Mc Clements DJ. Functional Materials in Food Nanotechnology. Journal of Food Science. 2006;71:107R-116R.

4. Smith MW, Laura J Martin. Probiotics and Prebiotics: Ask the Nutritionist. WebMD, USA. 2012

5. Abad CL, Safdar N. The Lactobacillus Probiotics in the Treatment or Prevention of Urogential Infections-A systematic Review. J Chemother. 2009;21(3):243-252.

6. Ooi LG, Liong MT. Cholesterol-Lowering Effects of Probiotics and Prebiotics: A Review of in Vivo and in Vitro Findings. Int J Mol Sci. 2010;11(6):2499-2522.

7. Mirjani R, Faramarzi MA, Sharifzadeh M, et al. Biosynthesis of tellurium nanoparticles by Lactobacillus plantarum and the effect of nanoparticle enriched probiotics on the lipid profiles of mice. IET Nanobiotechnol. 2015;9(5):300-305.

8. Waigankar Santosh S, Vimal Patel. Role of Probiotics in Urogenital Healthcare. $J$ of Mid-Life Health. 2011;2(1):5-10.

9. Tuomola E, Crittenden R, Playne M, et al. Quality assurance criteria for probiotic bacteria. Am J Clin Nutr. 2001;73(Suppl 2):393S-398S.

10. Araya M, Morelli L, Reid G, et al. Guidelines for the evaluation of probiotics in foods. Food and Agriculture Organization of the United Nations-World Health Organization, Geneva, Switzerland. 2002.

11. Harbolic Betty. Probiotics. Medicine Net, USA. 2015.

12. Wood C. Berkeley's Sugarlogix is engineering a sugar that's good for you. 2017.

13. Ben Xiao-Ming, Li J, Feng ZT, et al. Low Level of GalactoOligosaccharide in Infant Formula Stimulates Growth of Intestinal Bifidobacteria and Lactobacilli. World Journal of Gastroenterology : WJG. 2008;14(42):6564-6568.

14. Reardon Sara. Gut-brain link grabs neuroscientists. Nature International Weekly Journal of Science. Nature Publishing Group, a division of Macmillan Publishers Limited, USA. 2017.

15. Hsiao EY, McBride SW, Hsien S, et al. The microbiota modulates gut physiology and behavioral abnormalities associated with autism. Cell. 2013;155(7):1451-1463

16. Yuzhen W, Jiming X, Yunxu L, et al. Probiotic Lactobacillus casei Zhang reduces pro-inflammatory cytokine production and hepatic inflammation in rat model of acute liver failure. Eur J Nutr. 2016;55(2):821.

17. Rastall RA, Gibson GR, Gill HS, et al. Modulation of the microbial ecology of the human colon by probiotics, prebiotics and synbiotics to enhance human health: An overview of enabling science and potential applications. FEMS Microbiol Ecol. 2005;52(2):145-152. 
18. Sekhon, Bhupinder S. Food Nanotechnology - an Overview Nanotechnol Sci Appl. 2010;3:1-15.

19. Gbassi GK, Vandamme T. Probiotic Encapsulation Technology: From Microencapsulation to Release into the Gut. Pharmaceutics. 2012;4(1):149-163.

20. Huang Q, Yu, H, Ru Q. Bioavailability and Delivery of Nutraceuticals Using Nanotechnology. Journal of Food Science. 2010;75:50R-57R.

21. Lin W, Hong J-L, Shen G, et al. Pharmacokinetics of Dietary Cancer Chemopreventive Compound Dibenzoylmethane in the Rats and Impacts of Nanoemulsion and Genetic knockout of Nrf2 on its Disposition. Biopharm Drug Dispos. 2011;32(2):65-75.

22. Vidhyalakshmi R, Bhakyaraj R, Subhasree RS. Encapsulation "The future of probiotics": A review. Adv Biol Res. 2009;39:96-103.

23. Salmerón I. Fermented cereal beverages: from probiotic, prebiotic and synbiotic towards Nanoscience designed healthy drinks. Lett Appl Microbiol. 2017;65:114-124.

24. Uccello M, Malaguarnera G, Basile F, et al. Potential role of probiotics on colorectal cancer prevention. BMC Surg. 2012;12(Suppl 1):1S:35S.

25. Liang XJ, Chen C, Zhao Y, et al. Circumventing Tumor Resistance to Chemotherapy by Nanotechnology. Methods mol bio. 2010;596:467-488.
26. Liong MT. Roles of Probiotics and Prebiotics in Colon Cancer Prevention: Postulated Mechanisms and In-vivo Evidence. Int $\mathrm{J}$ Mol Sci. 2008;9(5):854-863.

27. Singh J, Rivenson A, Tomita $\mathrm{M}$, et al. Bifidobacterium longum, a lactic acid-producing intestinal bacterium inhibits colon cancer and modulates the intermediate biomarkers of colon carcinogenesis. Carcinogenesis. 1997; 18:833-841.

28. Okawa T, Niibe H, Arai T, et al. Effect of $1 \mathrm{c} 9018$ combined with radiation therapy on carcinoma of the uterine cervix. A phase iii, multicenter, randomized, controlled study. Cancer. 1993;72:1949-1954.

29. Zhang XB, Ohta Y. Binding of mutagens by fractions of the cell wall skeleton of lactic acid bacteria on mutagens. J Dairy Sci. 1991;74(5):1477-1481.

30. Larissa K, Eric C, Didier D Patrick C. Nanotechnologies for the treatment of colon cancer: From old drugs to new hope. International Journal of Pharmaceutics. 2016;514(1):24-40.

31. Katan MB, De Roos NM. Toward Evidence-Based Health Claims for Foods. Science. 2003;299(5604):206-207.

32. Katan MB. Health claims for functional foods: Regulations vary between countries and often permit vague claims. BMJ. 2004;328(7433):180-181. 